\title{
THE POSSIBILITY OF A NEW METAPHYSICS FOR QUANTUM MECHANICS FROM MEINONG'S THEORY OF OBJECTS
}

\author{
Matías Graffigna \\ Universidad de Buenos Aires, Argentina \\ E-mail: matiasgraffigna@gmail. com
}

\begin{abstract}
According to de Ronde it was Bohr's interpretation of Quantum Mechanics (QM) which closed the possibility of understanding physical reality beyond the realm of the actual, so establishing the Orthodox Line of Research. In this sense, it is not the task of any physical theory to look beyond the language and metaphysics supposed by classical physics, in order to account for what QM describes. If one wishes to maintain a realist position (though not nave) regarding physical theories, one seems then to be trapped by an array of concepts that do not allow to understand the main principles involved in the most successful physical theory thus far, mainly: the quantum postulate, the principle of indetermination and the superposition principle. If de Ronde is right in proposing QM can only be completed as a physical theory by the introduction of 'new concepts' that admit as real a domain beyond actuality, then a new ontology that goes beyond Aristotelian and Newtonian actualism is needed. It was already in the early 20 th century that misunderstood philosopher Alexius von Meinong proposed a Theory of Objects that admits a domain of being beyond existence-actuality. Member of the so called 'School of Brentano', Meinong's concerns were oriented to provide an ontology of everything that can be thought of, and at the same time an intentionality theory of how objects are thought of. I wish to argue that in Meinong's theory of objects we find the rudiments of the ontology and the intentionality theory we need to account for QM's basic principles: mainly the possibility of predicating properties of non-entities, or in other words, the possibility of objectively describing a domain of what is, that is different from the domain of actual existence.
\end{abstract}

\section{Introduction}

The wonder of Newtonian physics has many facets. It is not only a 'useful' theory we can apply to calculate velocities, accelerations, falls; it is not only a theory that allows us to perform experiments or to pose questions about how things are, given some conditions, or how things would be, given some others. It is also, and maybe mainly, a theory that allows us to understand 
the world in which we live in. Of course, not all physicists or philosophers are realists, who commit themselves to the thesis that holds that the terms of the theory actually refer to independent entities in the world. But, beyond the realism debate, I believe it to be without doubt that classical mechanics is a complete theory that allows us to understand reality, by offering certain concepts that seem to describe the macroscopic world we inhabit. Even if there is no independent reality where Newtonian particles collide and interact, that picture of the world seems to hold true when we inquire into certain domains of nature. So strong is said picture of the world, that it seems impossible to think beyond the concepts of Newtonian physics.

It is in this sense, I believe, that Quantum Mechanics (QM) is said to lack a consensus regarding its interpretation. What does it mean, for a theory that possesses a successful mathematical formalism, and unprecedented levels of empirical adequacy, to be without a unanimous interpretation? This means, basically, that "if we are to understand QM as a physical theory, and not merely as a mathematical or algorithmic structure, it is clear that we still need to provide a link between the mathematical structure and a set of physical concepts which are capable of providing a coherent account of quantum phenomena" (de Ronde, 2015A:8). In other words, we do not know what the theory is about. QM has a rigorous formalism, empirical adequacy, and outstanding technological applications, but it lacks still those concepts that allow us to form a picture of the world, to think about the 'reality' described by the theory, to do physics in the most complete sense. There is agreement in the literature, regarding the fact that QM possesses indeed a successful formalism and adequate experimental arrangements, even though we do not have the appropriate concepts to account for all of this:

"(1) The only consensual part of the theory is a formal skeleton enabling one to calculate the probability of various experimental outcomes at any time, given the initial preparation (Peres, 1995; Schwinger, 2001). (2) This formal skeleton is often complemented with bits and pieces of former pictures of the world borrowed from classical physics, but connected to one another in an unfamiliar and unruly way. A recurring complaint is that, as long as we are left without any truly coherent representation of the world and of its 'ontological furniture' compatible with the quantum formalism, we cannot claim that we truly "understand' quantum mechanics" (Bitbol, 2010:54-55) 
"Quantum mechanics brilliantly succeeds as a mathematical formalism: the numbers it provides are always successfully compared with experimental results. But it is often said to fail as an explanatory theory allowing us to understand the laws of atomic processes" (Lurat, 2007:230).

"Scientific advances can significantly change our view of what the world is like, and one of the tasks of the philosophy of science is to take successful theories and tease out of them their broader implications for the nature of reality. Quantum mechanics, one of the most significant advances of twentieth century physics, is an obvious candidate for this task, but up till now efforts to understand its broader implications have been less successful than might have been hoped. The interpretation of quantum theory found in textbooks, which comes as close as anything to defining "standard" quantum mechanics, is widely regarded as quite unsatisfactory. Among philosophers of science this opinion is almost universal, and among practicing physicists it is widespread. It is but a slight exaggeration to say that the only physicists who are content with quantum theory as found in current textbooks are those who have never given the matter much thought, or at least have never had to teach the introductory course to questioning students who have not yet learned to 'shut up and calculate!"' (Griffiths, 2011:2).

"Regarding its formal structure we could say that quantum mechanics seems to be a 'finished theory'. In terms of empirical adequacy, it provides outstanding results, its mathematical structure - developed in the first three decades of the 20th century by people like Werner Heisenberg, Pascual Jordan, Max Born, Erwin Schrödinger and Paul Dirac - seems able to provide until now the adequate modeling to any experiment we can think of. However, apart from its fantastic accuracy, even today its physical interpretation remains an open problem. In the standard formulation, quantum mechanics assigns a quantum mechanical state to a system, but 'the state' has a meaning only in terms of the outcomes of the measurements performed and not in terms of 'something' which one can coherently relate to physical reality. It is not at all clear, apart from measurement outcomes, what is the referent of this quantum state, in particular, and of the formal structure, in general. If we are to ask too many questions, problems start to pop 
up and simple answers seem doomed to inconsistency" (de Ronde, 2011:9)

Regardless of this agreement concerning the lack of a proper conceptual scheme that would allow a comprehensive understanding of QM in terms of a 'physical reality' of some sort, one could very well argue that this is indeed a futile enterprise, one that should be abandoned in favor of a more pragmatic or instrumentalist approach. Of this opinion are, for instance, Fuchs \& Peres (2000:1): "Contrary to those desires, quantum theory does not describe physical reality. What it does is provide an algorithm for computing probabilities for the macroscopic events ('detector clicks') that are the consequences of our experimental interventions. This strict definition of the scope of quantum theory is the only interpretation ever needed, whether by experimenters or theorists". So, we could ask ourselves, why bother with finding concepts or an interpretation for a theory that is already providing everything it should?

It is inevitable, in order to answer such a question, to make explicit what one believes should be a physical or even a scientific theory. The instrumentalist approach simply decides to ignore the fact that the theory - might — 'lacks' something, and wishes to pursue and insist in the already achieved successes of the theory. The interpretative 'problems' of the theory, such as the measurement problem, the basis problem, non-locality, non-separability... and the list goes on, are only set aside, swept under the rug, to allow for the wonderful computations to carry on. To argue with a position that does not acknowledge the existence of a theoretical problem is a hard enterprise, given how the desiderata concerning a physical theory are so different from one another. To them, I can only ask: is that all? Are we to satisfy ourselves by claiming that the most successful scientific theory produced by mankind is nothing more than an algorithm to compute probabilities, with no reference whatsoever to physical reality? Can we really settle with a theory that has no comprehensive concepts, but opens questions regarding nature and being we would choose never to answer?

To all of those who believe 'no' is the best answer for the posed questions, and believe physics is more than an algorithm that computes the results of the experiments we ourselves have designed, to all of those who yet believe in some kind of physical reality to be known by human scientific endeavor, a long road of problems lies ahead. I believe QM lacks concepts that would allow us to comprehend the reality which its formalism already describes, in the strong sense that implies that we need to find these concepts. For the unconvinced instrumentalist who remains happy computing, the remainder 
of this article will seem pointless. To the one who shares the desideratum of comprehending reality through physics, we need now to inquire into how this could be approached.

I will begin by presenting a map of possible interpretations for QM and I will argue in favor of the line of interpretations that states the need to find new concepts for QM. In section 3, I will offer a brief presentation of Meinong's theory of objects, which I will apply, in section 4, to some of the problematic issues of QM.

\section{The interpretation of $\mathrm{QM}$}

The quest of conceptually comprehending QM until today can be presented, following de Ronde (2011), in two main lines of inquiry: first, the tradition that beginning with Bohr has tried to comprehend QM based on classical concepts and has tried to make the formalism compatible with basic classical metaphysical principles; and second, another line of inquiry which attempts to take the successful formalism as a starting point and so, tries to find the appropriate metaphysical principles that would account for it:

"We believe that an interesting distinction that can help us to understand the huge interpretational map of quantum mechanics relates to the position one takes with respect to metaphysics. This controversial relation between physics and metaphysics displaces the problem of truth to a secondary stage and concentrates its analysis in the conditions of possibility to access and distinguish physical phenomena. Metaphysical schemes provide the coordinates through which the representational map of realistic stances can be developed. Among those who attempt to provide a metaphysical account of quantum mechanics there is a first group that tries, in different ways, to 'restore a classical way of thinking about what there is'. Staying close to at least some of the classical notions of physics (space-time, causality, objects, etc.) these approaches have no problem to give up the orthodox formulation of quantum mechanics. A second group also interested in the metaphysical question regarding quantum mechanics attempts to begin 'right from the start' with the successful mathematical formalism in its orthodox form, trying to learn about its structure and internal features in order to find a metaphysical scheme which is able to fit the formalism. We might consider the first group as going from metaphysics into the formal structure while the second group goes from the for- 
mal structure into the metaphysical scheme" (de Ronde, 2011:54).

The first path can be characterized, then, as that which attempts to comprehend the new theory, QM, with the old concepts, the classical Aristotelian-Newtonian ones. Bohr himself stated that "the unambiguous interpretation of any measurement must be essentially framed in terms of classical physical theories, and we may say that in this sense the language of Newton and Maxwell will remain the language of physicists for all time" (Bohr, quoted in de Ronde, 2011: 7). Probably one of the most famous examples in this line of inquiry is Bohm's hidden variable program, which according to de Ronde, "is forced to change the formalism with seemingly ad hoc moves; moves which can be only justified in relation to the prior metaphysical commitments" (de Ronde, 2011:54-5).

The Bohr-inspired program that seeks to find an interpretation for QM in the concepts and language of classical mechanics finds, among other approaches, its philosophical grounds in an interpretation of Kantian transcendental philosophy. Following Pringe's interpretation, the general idea is that the limits of possible experience require that a phenomenon be constituted both through sensibility and understanding, that is, through empirical intuitions and a priori concepts. Anything that falls out of these limits is considered by Kant to be metaphysical and not subject to scientific inquiry, inasmuch as it is beyond possible experience. According to Kant, certain a priori conditions must be met in order for something to be an object of possible experience: sensibility must provide the necessary empirical intuitions which are synthesized by the faculty of understanding according to the categories table. One of the most important elements in this table is the concept of causality. So, according to Pringe's Kantian reading of Bohr's interpretation of QM:

"If the quantum postulate is assumed, all pretension of reaching a spatial-temporal representation, which is at the same time causal, of an object subject to the postulate, must be abandoned. That is, if an object is within the domain of validity of the postulate, it won't be possible — as it is in classical physics - to synthesize the set of contingent data of a measurement, according to the concept of cause, as the effect of said object, representing this in space and time, in such a way that its states modify each other causally" (Pringe, 2012:183).

This means that the quantum postulate forbids 'quantum objects' to be objects of possible experience, inasmuch as it is not possible to synthesize 
the multiplicity of empirical data following the concept of causality. The main problem of this conclusion lies in the fact that objectivity is then lost for the quantum domain. So, how does QM remain a scientific theory, given that it violates Kantian transcendental conditions of validity?

The Bohrian answer to this question, as it is known, is contextuality. Within each experimental arrangement or measurement process, Bohr argues, we can synthesize the given objects, meeting thus the necessary conditions. The problem is, we cannot give a coherent account of the results of multiple experiments, since these immediately become incompatible in terms of the Kantian categories. Pringe goes on to argue that quantum phenomena must be described in classical terms, thus guaranteeing Kantian transcendental conditions.

"In effect, quantum phenomena are contextual, given that their validity is restricted to a determined type of experimental arrangement, and they are complementary, inasmuch as they mutually exclude one another; but at the same time, they are all necessary to account for experimental evidence. We face, then, a multiplicity of phenomena, whose objective character is established, but they do not yet acquire systematic unity" (Pringe, 2012:188-9).

This systematic unity is, of course, a necessary condition for scientific knowledge. So, quantum phenomena are given in terms of classical concepts which are referred to specific and distinct experimental arrangements. Now, each quantum phenomenon, from each arrangement, is incompatible, in classical terms, with each other (in most of the cases). Though incompatible, these results are mutually complementary, in the sense that they are all necessary to account for the empirical data that the theory produces. So, how can all these mutually incompatible and complementary results be brought to systematic unity in order to guarantee the objective validity of scientific knowledge?

"[...] Bohr distinguishes quantum objects from quantum phenomena, which are nothing more, than classical descriptions, whose totality exhausts the available information regarding the firsts. The systematic unity of quantum phenomena will only be reached when they are subsumed under the concept of quantum object. [...] The concept of a certain quantum object or system contains the representation of its state, and with it, the information about the different probabilities of the different results of the possible measurements that can be realized on the system. In this way, the 
multiple quantum phenomena are unified by a probabilistic law. Given a certain quantum phenomenon, the representation of the state of the quantum object establishes the probability of each and every phenomena of the object. So, the multiplicity of phenomena is synthesized through the concept of the object and subsumed under it. This synthesis allows, then, to carry out predictions such that, given a certain phenomenon, the probabilities of the different results of possible measurements are calculated based on the so called 'wave function' of the system" (Pringe, 2012:189).

The wave function then plays the role of the quantum object, which can never be directly given into intuition neither can it be synthesized according to the categories, but operates as the regulative systematic unity of the different quantum phenomena which are in fact, given to intuition and synthesized. The objective validity of QM is then grounded on the objective validity of classical physics, the reason for this being that classical concepts are the only ones that can attain objective validity. I shall quote Pringe in extenso one last time to appreciate the conclusion of such an analysis:

"In the first place, the objective validity of a classical object consists in its synthetic function of an empirical multiple, thanks to which, the intuitive representation of an object is constituted. On the contrary, the objective validity of the concept of a quantum object is based rather in its regulative task to provide systematic unity to the complementary phenomena (whose objectivity is guaranteed by the use of classical concepts to interpret the experimental results).

In second place, the concept of a classical object acquires objective reality when a given empirical multiplicity is subsumed under the concept thanks to the mediation of a scheme. So, the concept is exhibited directly in intuition. On the contrary, as we have seen, as a consequence of the quantum postulate, the conditions under which an empirical multiplicity is given, which should be synthesized by the concept of a quantum object, are incompatible with those conditions under which the concept can be applied. Therefore, a direct exhibition of such a concept in intuition is not possible. The concept of a quantum object acquires objective reality, rather, through an indirect exhibition in intuition, carried out through symbolic analogies" (Pringe, 2012:192-3) 
We can now appreciate what it means, in philosophical terms, that quantum mechanics can only be interpreted in terms of classical physics. These Bohrian declarations can be grounded in Kantian transcendental philosophy. What it means to be able to constitute an object is incompatible with quantum theory. Thus, all that is left for QM is to settle with classical representations, mutually incompatible, but mutually complementary. There is no quantum object we can constitute, but this concept operates not in a constitutive manner, but a regulative one, providing systematic unity to the multiple phenomena, presented in classical terms, through symbolic analogies.

In other words, we could say that the Bohrian interpretation of QM that seeks to understand QM in terms of classical concepts is right, if Kantian transcendental philosophy is also right. That is, it makes no sense to pursue new constitutive concepts for QM if the limits established in Kant's Critique of Pure Reason are indeed the a priori limits of what can be constituted by human thinking. All that is left is the possibility of finding metaphysical concepts, which would perform a regulative role, but not a constitutive one. This is, clearly, one possibility: but it implies that we must always find objective validity for these concepts, as Pringe says, 'indirectly'. Now, of course, we must ask ourselves, why should we trust Kant? The most direct way to 'refute' Kant's limits to human experience would be to find new limits to experience that are compatible with the quantum principles. But, again, why seek them if one thinks Kant is right about them? It would seem we need further motivation to enter such an enterprise.

It can be argued that we find in Kant the philosophical grounding for classical mechanics*. The space, which is the empty form of sensibility according to Kant, is the space of Euclidean geometry, which is, at the same time, the absolute space of Newtonian physics. The pure concept of causality, under which we synthesize phenomena and constitute objects of experience according to Kant, is the concept of causality that is needed for classical mechanics' descriptions of macroscopic interactions between bodies. These could be the transcendental limits of human experience. But they could also very well be the transcendental limits of classical experience. Why should these be the limits of all human experience? One may

\footnotetext{
*Regardless of whether or not Kant took Newtonian mechanics and Euclidean geometry as starting points for his theory, and regardless of whether or not these principles are needed for Kantian philosophy, the truth is that they seem highly compatible and that history, specially neo-kantism, has taken Kantian philosophy as a transcendental fundament for physics.
} 
argue that both the appearance of Relativity Theory and QM are sufficient reason to believe that we need new limits for human experience ${ }^{\dagger}$. We need a new ontology that is not grounded on classical metaphysical principles.

Another approach to a Kantian interpretation of QM is found in Michael Bitbol's work. The French author wishes not to accept the fixed given limits of experience developed by Kant, but to embrace his 'reflective metaphysical program' in order to analyze the different problems that arise from quantum theory. In this sense, the task is not to limit experience to Kant's words, but to inquire once again into the limits of human experience, based on the new developments brought about by QM:

"Kant's motto is that, despite its stemming from the "extravagant claims of speculative reason" (Kant, 1997, Introduction), metaphysics should not be rejected but disciplined. It should be given an epistemological rather than ontological status, so much so that ontology itself is seen as an epistemological tool. At the very end of Kant's work of reconstruction, metaphysical statements are then no longer seen as representations of something "out there", but as rules in a grammatical pre-ordering of experience. [...]Hence, metaphysics becomes nothing else than a reflective analysis of the powers and credence of reason" (Bitbol, 2010:59).

In this way, metaphysics is not seen as an objective description of an independently existing reality, but as a way to determine the possibility of knowledge. Given QM's 'new knowledge', one might argue, we need metaphysics to establish its conditions of possibility:

"One can thus adopt a pragmatic definition of the a priori instead of a purely intellectual one (Pihlstrm, 2003). According to this definition, an a priori form is no longer a universally necessary intellectual condition for objective knowledge, but a pragmatic condition locally and provisionally necessary for the determination of some intersubjectively shared domain of experimental or technological intervention" (Bitbol, 2010:62).

So Kant's all-limiting a priori becomes, under this new perspective, a contextual limitation to specific cases of knowledge. The task of a reflective metaphysics is not any more, then, to establish the limits of possible

† Of course, no a posteriori theory or evidence could refute a priori arguments. The point is that now, there seems to be reason enough to believe that those limits set by Kant are indeed to narrow. 
knowledge for all human cognitive activity, but rather, to describe the $a$ priori elements that are at stake in each context.

"This being granted, a solution (or rather dissolution) of the measurement problem boils down to finding a way to articulate the indefinite chain of relational statements of the quantum theory to the absolute statements that are used in experimental work. An articulation of this kind can easily be found, provided one realizes that the latter absolute statements are in fact indexical; provided one realizes that these statements are only 'absolute' relative to us, to our scale, to the open community of experimenters to which we belong (Rovelli, 1996 ; Bitbol, 2008). At this point, one is bound to realize the ineliminability of situatedness from the apparently neutral descriptions of quantum mechanics, and to accomplish thereby the reflective move typical of Kant's renewed definition of metaphysics" (Bitbol, 2010:75).

So the measurement problem is 'dissolved' because we come to the understanding that the 'absolute statements' of QM are in fact relative statements, the term of the relation being the community of scientists. The 'pragmatic a priori' means nothing else than the explicitation of the metaphysical principles that underlie each experimental arrangement. Since each of these is in fact produced by the community itself, all that remains is to acknowledge this fact and consider QM as interpreted in our own terms:

"But in quantum physics, no event should be ascribed autonomy. In this case, every event is tantamount to an observable value ascription, and an observable is only defined relative to an effective instrumental possibility of assessing it. In quantum physics, the instrumental context is not only a way of getting access to an event; it is a way of generating it" (Bitbol, 2010:78).

So, according to this pragmatic a priori, we are to settle ourselves with no more than the conditions of possibility of a given situation, which coincides with the fact that we determine ourselves the conditions for a given experimental arrangement. There is nothing beyond that situation and the so called 'paradoxes' of QM are dissolved inasmuch as they no longer constitute a problem, if we accept that each measurement is situated:

"This represents a major difference with classical physics. In classical physics, the simple truth that we act as situated subjects of knowledge could be bracketed, and a naturalized description of 
the world including ourselves taken as objects could pretend to be universal. Instead, quantum physics manifests the bounds of this attitude of all-pervasive naturalization. It makes one realize that the irreducible fact of situatedness is a necessary presupposition of objective knowledge and cannot thus be objectified itself. This, of course, was pointed out by many generations of transcendental philosophers, from Kant to Husserl and beyond; but quantum physics leaves little room for those who want to ignore their lesson" (Bitbol, 2008:212).

In my opinion, Bitbol's so called transcendental interpretation of QM boils down to a sophisticated defense of an instrumentalist position. To ascribe Husserl or Kant such a conception is as fair as believing we have come any closer to an understanding of the problems involved in QM, because we call "pragmatic a priori" the renounce of a realist program for QM. Kant believed that the a priori concepts of pure understanding referred to actual, existing, independent reality, by way of the empirical intuitions that are synthesized under such concepts. Husserl believed that the constitution of phenomena in natural attitude is guided by the world itself, and that in ultimate stance, the question about how phenomena are constituted is the question as to why the subjective constitution of phenomena is valid, in the sense that it corresponds with the reality 'out there'. The whole point of the transcendental question into the conditions of possible experience is to determine in a universal manner how it is that we know the world. There is nothing transcendental, in any relevant sense, in the claim that we generate an instrumental context each time we perform an experiment.

Pringe's reading of Bohr's interpretation is based on a solid understanding of Kantian philosophy. My only criticism to it is that, while Kant's philosophy successfully grounds in transcendental conditions classical mechanics, it fails to bring us any closer to an understanding of QM and closes the door for any project that seeks to really empower QM by acknowledging that the success of the theory should be taken seriously. We cannot understand QM, know what the theory is talking about, if we try to force it into old schemes, and settle with 'symbolic analogies'. The question of what does QM talk about, needs to be taken seriously, instead of trying to explain why the question cannot be answered.

On the other hand, Bitbol's position falls short of being transcendental or realist in any relevant sense. If anything, it is an elaborate account of the claim that QM needs no interpretation: because we are situated, we cannot escape our situation, and therefore must settle with a contextual reading 
of the results of QM, that denies them any kind of autonomy, validity and even reality.

Let us now move into the second group of interpretations of QM, that which wishes to find the proper metaphysical principles for QM taking as a starting point the successful formalism, and which wishes to do so not in instrumentalist terms, neither in nave realist terms, but in the sort of realism that takes into consideration the fact that scientific theories represent reality, a reality that exists out there, but that we can only access through a certain array of concepts. De Ronde calls this a "Representational Realist Stance", and defines it as follows:

"A representational realist account of a physical theory must be capable of providing a physical (and metaphysical) representation of reality in terms of a network of concepts which coherently relates to the mathematical formalism of the theory and allows to make predictions of a definite field of phenomena (expressed through such concepts)" (de Ronde, 2015:12-3).

We can take, then, the realist stance and the search for a new ontology of QM as two fundamental desiderata in the quest of interpreting QM. If we do so, we can better see what the problem is with interpretations that still seek to keep the classical concepts for QM. The argument is simple: it is the theory which tells us how to understand reality and what is and is not out there. Physical theories are based on metaphysical principles which are adopted without question and, of course, without possible scientific justification, since they are the basic principles upon which the concepts of the scientific theory are developed. In the case of physical theory, de Ronde argues, we are still trapped by Aristotle's basic metaphysical principles: the Principle of Existence, which determines that an entity, that which exists, can only do so only in spatio-temporal way; the Principle of NonContradiction, which forbids the attribution of contradictory properties to anything that exists, since it assumes that reality is in itself of a noncontradictory nature; and the Principle of Identity, which asserts that an entity is identical to itself, and that its essential properties are maintained through time. The basic assumption in Aristotelian metaphysics and later in Newtonian, taken to the extreme, is that everything that is, all that exists, can only do so for real, in actuality. In other words, there is only one real existent mode of being: the mode of actuality:

"The general metaphysical principle implied by the understanding of Newtonian mechanics, that 'Actuality = Reality', has become an 
unquestionable dogma within physics. As a silent fundament all of physics has been developed following the metaphysics of actuality. And even though QM was born from a deep positivist deconstruction of the a priori classical Newtonian notions -and in this sense the philosophy of Mach can be understood as the very precondition for the creation of both QM and relativity theory- it was very soon reestablished within the limits of classical metaphysics itself. The constrains of actuality have been unquestionably accepted by philosophers of physics either in terms of hic et nunc observation (empiricism and its variants) or as the mode of preexistence of properties (realism). Both positions have remained captive of actualism; trapped in the metaphysical net designed (through the PE, PNC and PI) by Aristotle around the 5th century before Christ and imposed by Newton in the 18th Century of our time. Actual (preexistent) properties and actual (here and now) observations are two sides of the same (metaphysical) coin" (de Ronde, 2015:20)

All attempts to understand QM have been precluded to do so, due to the limitations imposed by such a metaphysic, "But what if QM cannot be subsumed under the metaphysical equation imposed by Newtonian physics: Actuality = Reality?" (de Ronde, 2015:21-2). If that is the case, and it is the unquestioned presupposition that reality can only be in the mode of actuality which has prevented a successful interpretation of QM, then a new path is clear ahead:

"We need to develop a new way of understanding reality beyond the ruling of actuality. To escape the ruling of actuality — both in terms of hic et nunc observation and pre-existent propertiesmeans to abandon, on the one hand, the idea that we have a clear definition of what is observed according to QM, and on the other hand, the idea that actuality is the only possible way to conceive and understand physical reality. Our strategy is to take as a standpoint the formalism and its predictive power in order to develop new physical concepts which relate coherently to the formalism and can allow us to think about the physical meaning of quantum phenomena." (de Ronde, 2015:23-4).

The project then comes to light. To search for new concepts for QM means to develop a new ontology. A new ontology is not the same as new ontic categories. The task is not to expand the list of what there is, but to rethink the principles under which we claim that something is or can be. 
The principle that underlies all previous metaphysical endeavors and, therefore, all attempts to find a proper conceptualization of QM, seems to be the principle that equates reality to actuality. Two things are then needed to carry on forward: a new ontology that does not reduce reality to what is actual, and a new theory of experience that allows us to understand how such a domain of reality, which is not actual, could be thought of, experienced. In other words, we need a new ontology and 'a new' phenomenology.

\section{Meinong's Theory of Objects.}

In this section I wish to offer a schematic presentation of philosopher Alexius von Meinong's Gegestandtheorie or theory of objects. Based on the conclusion of the previous section regarding the need for a "new ontology" that would allow to fully grasp the principles of QM, I believe Meinong's ontology is a good place to start. Meinong is a disciple of Austrian philosopher Franz Brentano, who can be considered to have founded a philosophical school, the so called "School of Brentano", of which I wish to recover one main principle that appears clearly in Meinong's philosophy and is relevant for the present purposes ${ }^{\ddagger}$. The key point of Brentano, or at least of the 'Brentanian philosophers', is the correlation between the psychological and the ontological.

Brentano's reading of Aristotelian realism leads him to consider that there is a parallelism between mental acts and their objects, one the one hand, with objects 'in themselves', on the other. The crucial thesis is that those objects as they are, unlike Kant's nomena, are given to the mind and can be fully known. Thus, philosophy is the inquiry both into the mental acts and its correlates (psychology), and into objects and their nature as such (ontology). In the words of Barry Smith:

"Descriptive psychology, as Brentano here understands it, seems to consist precisely in a psychology that will issue in an ontologically sophisticated theory of the different types of parts, of such a sort that the specification of parts will be at the same time a specification of the ways in which these parts are fitted together into wholes" (Smith, 1994:47)

A psychological investigation, thus, would yield as results not only in-

\footnotetext{
¥For a comprehensive reading on the philosophy of Brentano and its disciples, see: Smith, Barry (1994), Austrian philosophy. The legacy of Franz Brentano, Open Court Publishing Company, Chicago and LaSalle, Illinois.
} 
formation regarding mental acts themselves and consciousness as such, but, inasmuch as investigating the mental correlates of mental acts is investigating objects, it would also yield the ontological information of the objects as they are.

Historically speaking, this parallelism found in Brentano's theory, was inherited by most (if not all) of his disciples, who later focused in different domains of inquiry. In the case of Meinong, the disciple who interests us here, this was translated into a theory of objects which is, as we will see in a moment, both a theory of objects as they can be thought of, as well as a theory of objects as they are or can be:

"For where Brentano applied his descriptive realist method almost exclusively in the area of psychology, his students extended it in systematic ways to other domains of inquiry. We can in fact distinguish in their work three branches of what might be called 'descriptive ontology': the ontology of things (or objects in the narrow sense), the ontology of states of affairs, and the ontology of values, a tripartite division which flows in an obvious way from Brentano's tripartite division of acts.

The ontology of things or objects arises when one turns from the psychology of presentation to an investigation of the nonpsychological correlates of presenting acts. 'Object' is then understood as: 'possible correlate of presentation'" (Smith, 1994:52).

In order to make clear how this tripartite division follows obviously, let me very briefly present Brentano's tripartite division of acts: all acts of consciousness are of one of the following type: an act of presentation, in which the object is simply present to the mind; an act of judging in which the object's existence is either affirmed or negated; and an act of interest, in which the object (both presented and usually judged to exist) is loved or hated $^{\S}$. It is in the first simple sense in which Meinong's theory of objects can be first understood: an object is that which can be thought of. As such, it must have some sort of being. Which 'type' or mode of being it has, must be 'decided' once we know more about that object we are thinking of.

This common principle of the School of Brentano is of crucial importance in the task to 'find new concepts' for QM. As it has been shown in the previous section, especially considering Pringe's Kantian interpretation of Bohr, an ontology that does not allow us to comprehend how the ontologi-

$\S$ Cf. Smith (1994:42-4) 
cal domain to which 'quantum objects' belong, can be experienced, would not be a very fruitful enterprise. In this sense, the typical psychologicalontological parallelism of the School of Brentano should prove interesting: it is not only a theory about what there is (ontology) what we seek, but also a theory about how what is can be thought of (psychology). The problem with the Kantian approach is that it limits experience only to the domain of what can be empirically intuited, thus closing both ontology and psychology to very limited possibilities, and leaving everything else outside of the 'scientific' knowable world and relegated to mere metaphysical speculation.

It is important to make a distinction between an object of possible experience and an object that plays a role in experience. From the Kantian perspective, we only have experience of spatio-temporal objects, yet, there are other objects, such as regulative objects or metaphysical objects, which cannot be experienced, but perform a function in guiding or regulating experience. Such objects are, for instance, God, the Soul or even Truth, understood as a regulative idea. The problem arises with the idea that certain objects that we claim, belong to nature, such as QM-objects would be, are not objects of possible experience, and all they can do is regulate or guide our experience of classical objects, which, in its turn, would tell us something about the domain of QM. The accusation against Kant-BohrPringe is not that they find no role for these objects which cannot be experienced, the problem is the claim that there are, in physicial nature, objects which cannot be experienced and should perform a function similar to that which performs, for instance, the idea of God.

Let us now move into Meinong's theory. A good starting point for the present discussions is Meinong's 'prejudice in favor of the actual'. The idea is that the interest of inquiry has always been so focused on what exists in the sense in which spatio-temporal objects exists, that a whole domain of objects of knowledge has been left aside, objects which are in their own sense. A theory of objects, then, should focus not only in those objects that exist in such a manner, but in all objects that have some sort of being:

"If we remember how metaphysics has always been conceived as including in its subject matter the farthest and the nearest, the greatest and the smallest alike, we may be surprised to be told that metaphysics cannot take on such a task. It may sound strange to hear that metaphysics is not universal enough for a science of Objects, and hence cannot take on the task just formulated. For the intentions of metaphysics have been universal (a fact which has so often been disastrous to its success). Without doubt, metaphysics 
has to do with everything that exists. However, the totality of what exists, including what has existed and will exist, is infinitely small in comparison with the totality of the Objects of knowledge. This fact easily goes unnoticed, probably because the lively interest in reality which is part of our nature tends to favor that exaggeration which finds the non-real a mere nothing--or, more precisely, which finds the non-real to be something for which science has no application at all or at least no application of any worth" (Meinong, 1981:77)

Meinong's concern, then, can be said to exceed the preoccupation for what is 'real' or what exists, or that which metaphysics encompasses. Meinong is concerned with providing a theory that can account for all objects, i.e., everything that can be thought of. In this sense, there are more objects than 'things'. We can think of more things than those that actually exist. And in a very relevant sense, we can have experience of more objects than those that exist only in the form of spatio-temporal entitites.

We can approach Meinong's theory of objects by comparing it with Russell's theory of definite descriptions. Historically speaking, Russell can be considered the victor in a dispute between the two authors, regarding how to consider the attribution of properties to non-entities:

"Meinong was concerned with the problem of explaining the apparently correct attributions of properties to non-entities, especially in intensional contexts, and the closely related problem of intensionality. It is often said that Russell's theory of descriptions simply solved the problem of ascriptions of properties to non-entities; and this is usually supported by pointing to the Russellian analysis of non-existence claims as claims about entities" (Routley \& Routley, 1973:225).

Let us take the example of Pegasus and the corresponding statement "Pegasus does not exist". According to Russell, since logic and hence all predication is always extensional, no statement can correctly be predicated of a logical subject that does not exist. So, the statement about Pegasus should be correctly paraphrased into "The class of existing items does not include Pegasus". We can see here how the second statement does not have Pegasus as its logical subject, but the set of existing things. Given that Pegasus does not exist, I cannot correctly attribute it with any property at all, not even non-existence. Now, the consequence of the Russellian approach is that I cannot predicate anything at all of a non-existing entity. Thus, 
the statement "Pegasus is a winged horse", given how there is no Pegasus, must be considered false, the same as the statement "Pegasus is identical to Pegasus". This 'solution' offered by Russell and accepted by the analytic tradition of philosophy of language has as a consequence the impossibility of predicating of anything that does not exist. Now, this logical maneuver carried out by Russell can, of course, be considered legitimate and suiting very specific purposes. It goes along great, for example, with a positivist ontology as the one assumed nowadays, but it falls short for pretty much any other purpose:

"While such a 'solution' to the problem of the attribution of properties to non-entities might be satisfactory for a few limited purposes, for many purposes it is not. Russell's theory does not even begin to provide a solution to the main problems which concerned Meinong, viz. that of obtaining a satisfactory account and explanation of truth (or factuality) in intensional discourse, and of the logical behaviour of subjects, descriptions and quantified expressions in intensional discourse, of explaining the apparent truth of some statements about non-entities and the peculiarity or falsity of others, and of obtaining a non-Platonistic account of mathematics." (Routley \& Routley, 1973:226)

Meinong's solution to this problem runs in a very different line. Rather than denying the possibility of attributing properties to non-entities, Meinong proposes quite a different principle to start from, the so called "principle of independence of being-so (Sosein) from being (Sein)" and it states as follows: "The Object is by nature indifferent to being, although at least one of its two Objectives of being, the Object's being or non-being, subsist" (Meinong, 1960: 82 and 86 respectively). We can know an object, we can think about it, predicate of it, without yet knowing whether or not the object exists. Things are said to exist when they do so in space-time. In this sense, all of mathematics is able to predicate from objects that do not exist.

An object is a simple unit; it can be a part of an objective, which is Meinong's term for what is usually known as a state of affairs, a complex 'situation'. For example, the golden mountain is an object, "the golden mountain is made of gold" is an objective. The golden mountain does not exist, yet the just stated objective subsists. "Why doesn't the golden mountain exist?", one might ask. And Meinong's answer would be "because it's made of gold and there are no such things as golden mountains". Yet we only 
know that golden mountains do not exist, because we understand what a golden mountain is, we can grasp the object 'golden mountain': "If I should be able to judge that a certain Object is not, then I appear to have had to grasp the Object in some way beforehand, in order to say anything about its not-being, or more precisely, in order to affirm or deny the ascription of non being to the Object" (Meinong, 1960:84).

Now, regarding certain other objects, such as the round square, we cannot attribute them with any kind of being. They are, in fact, Aussersein or outside being. Yet, for Meinong, unlike for Russell, round squares are indeed round and square, hence we know they are impossible objects. The objective "round squares are impossible figures" subsists. This objective has a being so, inasmuch as it subsists, even if the object, the round square, must be attributed with not-being. Compare this with Russell, for whom round squares are neither round nor square, since the class of existing items does not comprise such entities, nothing can be predicated of them with truth.

We can see the elements of Meinong's ontology in the following chart:

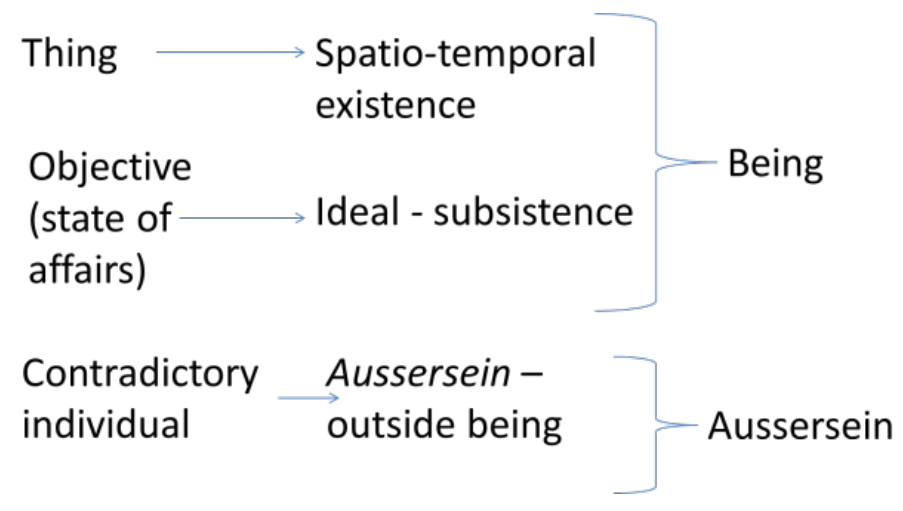

The most important consequence of Meinong's theory of objects, at least for our present purposes, is that it allows to know and describe objects without a prior commitment to its existence. The domain of being is expanded beyond existence, allowing for science to inquire into objects that do not exist in the way of spatio-temporal things, but rather have a different kind of being, they subsist:

"The first distinctive thesis of Meinong's theory is that very many objects do not exist in any way at all. Nevertheless we can make 
true statements in which such objects occur as proper subjects. Since the term 'object' carries no existential commitment, the standard attempt to represent Meinong's theory as a Platonistic theory or as a levels-of-existence theory is, on the face of it, seriously mistaken in the case of ground-floor objects at least. Thus one very important feature which Meinong's theory shares with any thoroughgoing and genuinely non-existential logic is the rejection of the Ontological Assumption (OA), the view embodied in all standard modern logical theories and most empiricist theories (e.g. Hume), that one cannot make true statements about what does not exist. Alternatively, the OA is the thesis that a non-entity cannot be the proper subject of a true statement (where the proper subject contrasts with the apparent subject which is eliminated under analysis into canonical form). The OA was explicitly rejected in Meinong's Independence Thesis (IT) stating the independence of Sosein from Sein: according to the thesis an item's having properties does not imply its existence" (Routley \& Routley, 1973:227).

I'd like to offer a brief presentation of two more concepts that are central to Meinong's theory of object and could prove useful in the following section. The first one is the concept of incomplete object. A complete object is, paradigmatically, a thing, a spatio-temporal entity. They are complete both in the ontological and gnoseological senses. An object is complete when it is completely determined in all its properties regarding all its relations with all other objects. In other words, a complete object is the one that has a determined answered for all questions posed in terms of the law of the excluded middle. From the gnoseological side, a complete object is that of which I can know all is properties, all its determinations. This glass of water next to me, for instance, is a complete object. When I ask "is it transparent?" I answer "yes", "is it $10 \mathrm{~cm}$ tall?", yes... and so on. The object is determined in every sense, even if I do not know all of its properties, in principle I could, or if I don't it is due to empirical reasons and not due to a natural impediment of the object.

An incomplete object, on the other hand, is that which is not determined regarding all of its properties. This means, not only that one does not know these attributes, but that indeed the object is, in an ontological sense, undetermined. Excluded middle does not apply to it. For example, the abstract circle described by an Euclidean geometry is not determined as to its size, its color, its texture, its location, etc. It is not that these properties are unknown, they are simply not in the object. I could not know, for 
example, that the circle's area is $\pi \cdot r^{2}$, yet the object is determined in this respect, whereas the color of the circle is not something unknown, but undetermined. It is just a property that the abstract geometrical circle does not possess.

An object that is either determined or undetermined will also be (un)determined in its mode of being:

"An object which is completely determined in its Being-So ( $S o$ sein), is also completely determined in its mode of Being (Sein). Correspondingly that object which is incompletely determined in its Being-So, is also incompletely determined in its Being. Of the incomplete object $\mathrm{A}$, which is not determined as to its Being, it cannot be stated 'A is' or 'A is not'. It is still possible that it is and that it is not. Here again, possibility is a third alternative to the two contradictory factuality determinations and, moreover, something definitely positive. The indeterminateness of incomplete objects with respect to factuality of Being and Being-So is wholly compatible with their determinateness as to the possibility of Being and Being-So. Thus, though factuality cannot be attributed to incomplete objects, possibility may. The freedom of the incomplete objects from the law of the excluded middle enables them to be the 'carriers' (Trger) of 'pure possibilities"' (Michaelis, 1942:401).

Which brings us to the second and last concept I wish to introduce, that is, the concept of possibility. "Possibility is a quantitative property which can be intensified up to the limit of factuality" (Michaelis, 1942:397), meaning that possibility can be thought of as a line that goes from impossibility to factuality/actuality. Possibility is predicated not upon objects themselves, but upon objectives; one should not say "A is (not) possible", but "it is (not) possible that A". Then, in one extreme of the possibility line, we would find all the objectives that have as components completely undetermined objects, contradictory objects such as the round square. In 'the middle' of the line, we would find incomplete objects, such as mathematical entities or objects that are probabilistic in their own nature; until the other extreme of the line, where determined, complete objects, that is, spatio-temporal things, are found.

To conclude this section, the aim of which was no other than to offer a schematic presentation of some of the rudiments of Meinong's ontology in the hope they can be applied to some of the issues of QM in the following section, we can state that Meinong's theory of objects takes as a 
starting point the concept of object, that what can be thought of, and frees objects from the restraint of existence in the task of knowledge; meaning that there is a realm of being that goes beyond existence/actuality that can be scientifically known. Objectives can be composed both by real existing objects or inexistent objects. In both cases we say of objectives that they subsist. Finally, objects can be either complete or incomplete in the strong ontological sense, and depending on this, we will find them in one place or another of the possibility line, which again, is an ontological possibility and not an epistemic one.

\section{A Meinongian ontology for QM}

In this section I would like to apply some of the Meinongian concepts presented in the previous section, to some of the main interpretative problems in QM. In particular, I will address quantum superpositions.

Quantum superpositions, also known in the literature as "Schrödinger's cats" raise a series of difficulties that concern not only the discussions in foundations of QM, but also the theory itself. The problem regarding superpositions, according to (da Costa \& de Ronde, 2013), is that they seem to violate the principle of non-contradiction when establishing mutually exclusive terms as in for example $\alpha|\uparrow\rangle+\beta|\downarrow\rangle$. Moreover, a further problem appears at the time of determining what objective physical process is responsible for the measurement outcome of only one of the terms; this is known as the "measurement problem". These problems are yet without an accepted solution and, according to de Ronde, the way to solve them lies not within the insistence on the 'measurement problem', on trying to find a way to classically justify the measurement outcomes, which are always taken as a starting, legitimizing point, but rather, by looking into the superpositions themselves. All attempts are guided by the will to understand QM in classical terms, by ignoring that there are, in fact, superpositions as described by the quantum formalism, but also being used for the most diverse technological applications".

This misguided approach rests, among others, in one metaphysical supposition that is operating in every attempt to interpret QM, that is, that reality equals actuality. In other words, that something can only be considered as real, as 'truly existent', with ontological density, if and only if that something is in the mode of the actual. Actual here must be understood as

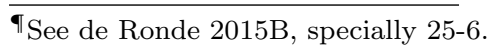


a metaphysical mode of being, that in which the object in question is completely determined in every respect, as it is the case of physical bodies in classical Newtonian physics. But, "if we are willing to discuss the possibility that 'Quantum Physical Reality Actuality', then there is plenty of space to interpret and represent quantum superpositions in terms of (non-actual) physical reality" (de Ronde, 2015B:3). What would it mean for "quantum physical reality' to be different from actuality?

An answer can be sought in Meinong's ontology, as I have presented it in the previous section. To begin with, we can consider any definition of a quantum superposition, as the one quoted above, to be a subsisting objective in the Meinongian sense. We mean by this two things. First, that the state of superposition as such should not be thought of as an object, but as an objective. Remember that objectives are, for Meinong, states of affairs, complex objects, made of objects. Hence, in $\alpha|\uparrow\rangle+\beta|\downarrow\rangle$ we find the terms $|\uparrow\rangle$ and $|\downarrow\rangle$ as being the objects that conform the whole objective. Second, by saying that the entire objective subsists, we are saying that it has a specific mode of being, that is not the mode of being of existent actual things, and we are also saying, that we need not worry (yet) about whether or not the objects that compose this objective, the terms in the equation, exist or not; because, as explained in the previous section, judgments about existence are independent of other judgments we can make about objectives.

Superpositions are contradictory only when thought from the perspective of what is actual. But we could understand the wave function, a complex objective which has several bases, some of which are states of superpositions, as being an incomplete object. Now, incomplete objects are undetermined not only in a subjective sense, in the sense that we don't know certain properties it has, but also in an ontological sense, that is, incomplete objects are indeed undetermined regarding many of its properties. This indetermination is only a problem, again, if we want the wave function to be a complete object and more precisely a spatio-temporal thing that exists in the same sense as Newtonian particles do. But are we not, in this way, calling for unnecessary problems? Why insist in a metaphysical principle, which as such cannot be demonstrated, if it turns out to make any coherent interpretation impossible?

According to Meinong possibility is a line that goes from impossibility to actuality. What if the domain that QM describes does not belong to the realm of actuality, but to that of pure possibility?: 


\section{Possibility}

$\begin{aligned} & \text { Aussersein: } \\ & \text { Objects } \\ & \text { outside being, } \\ & \begin{array}{l}\text { contradictory, } \\ \text { impossible }\end{array} \\ & \qquad \begin{array}{l}\text { Objectives: they subsist, they are } \\ \text { incomplete objects }\end{array}\end{aligned}$
$\begin{aligned} & \text { Objects known } \\ & \text { to be impossible }\end{aligned}$
$\begin{aligned} & \text { Mathematical objects } \\ & \text { Objects of an essentialy } \\ & \text { probabilistic nature }\end{aligned}$

A first consequence of this way of interpreting the problem would be that: the superpositions problems should be investigated separately from the measurement problem. By this I mean, one issue is understanding measurement in the sense of 'actualization' of quantum states, that is, one issue is the relation there is between the domain of reality described by QM and how that domain becomes actual in a measurement. But the wave function, as described by QM, should constitute an independent and legitimate problem for quantum theory, one that should be understood in terms of possibility, rather than actuality. Thinking of as an incomplete object allows to understand that there is nothing contradictory in the expression of a superposition state. At the same time, if 'real', in the sense of strong being, in the sense of 'what really is', is separated from what is actual, allowing to enlarge the domain of relevant being beyond actuality, then, the wave function, understood as an incomplete object, can be said to be in its own right, even if it doesn't exist in the sense of the actual. This being of the wave function, now in the mode of possibility rather than actuality, is from a Meinongian perspective a legitimate domain of being, in the relevant sense that it is a domain susceptible of scientific inquiry.

Following this line of thought, the primordial question should no longer be "how does nature decide the result of a measurement given a superposition?", but, rather, "what is a superposition and what can it do besides 
being actualized in a measurement?" It is not that the process of actualization through measurement were irrelevant or unimportant for QM, but it seems that some previous knowledge is necessary in order to tackle it. We do not yet fully understand what a superposition is, but we do have the formalism, and also the possibility of grounding this understanding in a different ontology from the one of classical Newtonian mechanics. By taking the realm of possibility seriously, and the independence of being-so from being, I believe, we can start seeking for new physical concepts that allow the proper comprehension.

We can seek for these concepts now, not in terms of complete determined actual classical objects, but with a different ontology. We can think of superpositions as incomplete objects. This change allows superpositions to bear "mutually contradictory properties" without collapsing understanding. The relevant question would be now, not how one term of the equation appears instead of the other in a measurement outcome, but, for instance, how do different superposition states, none of them actualized, become 'entangled' and interact with one another? If this occurs independently of a measurement process, as it seems to be the case, then we need to begin to understand that these curious states have objective physical properties that are entirely independent of actuality.

Superpositions and the wave function must be understood in their own right. This means we need to find the metaphysical principles that allow for their comprehension regardless of the domain of actuality. In this sense, de Ronde proposes to replace the classical Aristotelian metaphysical principles upon which classical physics are based, for the principles that spring from the quantum theory: instead of the Aristotelian principles of Existence (that being is being is the mode of the Aristotelian entity, as a space-time unity, a complete object in the Meinongian sense); of non-contradiction, and of identity (that is, the identity of an entity through time); instead of said principles, "This realm [the realm of QM] is defined by the principles of indetermination, superposition and difference" (de Ronde, 2015:26). This means that to understand what constitutes a quantum object; we need to define it according to the principles proper to the domain it belongs, the domain of QM, the domain of possibility; and not in terms of actuality.

\section{Conclusions}

I have argued in favor of a realist approach to the problem of the interpretation of QM and, following that line, I have offered some arguments as to why such problem should be sought to be solved from a perspective that 
prioritizes the quantum formalism and the elements proper of the theory that have led to so much experimental and technological success, over those orthodox interpretations that seek to maintain all physical understanding within the limits of classical physics. Having accepted then the need for new ontological concepts that could allow for such an interpretation, I have offered a brief presentation of Meinong's theory of objects. In particular, the concepts of subsistence, possibility and incomplete objects, open, I believe, the opportunity to consider that the wave function, as a sort of 'quantum object', belongs to a different mode of being than that of actuality. I do not claim to have found here any revolutionary results, but only to have offered some basic ontological considerations in order to guide the discussion in the foundations of QM. In this sense, I believe that Meinong's ontology allows for a scientific comprehension of objects that do not exist in the mode of the actual, and that these objects could be the objects of QM. If this is true, the only result I have to offer is an indication as to how to proceed in the quest to comprehend quantum reality: that is, not in its constant reference to measurements and actuality, but as a legitimate domain in itself.

\section{Acknowledgements}

I'd like to thank Christian de Ronde and Hernan Pringe for their careful reading of the manuscript and the valuable comments and insights they offered.

\section{References}

1. Bitbol, M. (2008), Consciousness, Situations, and the Measurement Problem of Quantum Mechanics, NeuroQuantology, Vol. 6, Issue 3, pp. 203-213

2. Bitbol, M. (2010), Reflective Metaphysics: Understanding Quantum Mechanics from a Kantian Standpoint, Philosophica N83, pp 53-83

3. De Ronde, C. (2011), The Contextual and Modal Character of Quantum Mechanics: A Formal and Philosophical Analysis in the Foundations of Physics, Print Partners Ipskamp, Enschede

4. De Ronde, C. (2015A), Probabilistic Knowledge as Objective Knowledge in Quantum Mechanics: Potential Powers Instead of Actual Properties

5. De Ronde, C. (2015B), Quantum Superpositions Do Exist! But 'Quantum Physical Reality Actuality' (Reply to Dieks and Griffiths)

6. Fuchs, C. and Peres, A. (2000), Quantum theory needs no 'interpretation', Physics Today, 53, 70

7. Griffiths, R. (2011), A Consistent Quantum Ontology

8. Lurat, F. (2007), Understanding Quantum Mechanics, en Rediscovering Phenomenology, Boi, L., Kerszberg, P. \& Patras, F. (eds), Springer, Dodrecht. 
9. Meinong, A. (1960), Theory of Objects, in Realism and the Background of Phenomenology, ed. Chisholm, R., Free Press.

10. Michaelis, A. (1942), The Conception of Possibility in Meinong's "Gegenstandstheorie", Philosophy and Phenomenological Research, Vol. 2, No. 3, pp. 394-403

11. Pringe, H. (2012), La filosofa trascendental y la interpretacin de Bohr de la teora cuntica, scienti zudia, So Paulo, v. 10, n. 1, p. 179-94

12. Routley, R. \& Routley V. (1972), Rehabilitating Meinong's Theory of Objects, Revue Internationale de Philosophie, Vol. 27, No. 104/105 (2/3), MEINONG (1973), pp. $224-254$

13. Russell, B. (1905), On Denoting, Mind, New Series, Vol. 14, No. 56, pp. 479-493

14. Smith, Barry (1994), Austrian philosophy. The legacy of Franz Brentano, Open Court Publishing Company, Chicago and LaSalle, Illinois 